\title{
Cones from quantum groups to tropical flag varieties
}

\author{
Xin Fang $^{1}\left(\mathbb{D} \cdot\right.$ Ghislain Fourier $^{2} \cdot$ Markus Reineke $^{3}$ \\ Received: 29 May 2020 / Accepted: 22 January 2021 / Published online: 9 February 2021 \\ (c) The Author(s) 2021
}

\section{Abstract}

We relate quantum degree cones, parametrizing PBW degenerations of quantized enveloping algebras, to (negative tight monomial) cones introduced by Lusztig in the study of monomials in canonical bases, to $K$-theoretic cones for quiver representations, and to some maximal prime cones in tropical flag varieties.

\section{Introduction}

The aim of this work is to point out a rather unexpected relation between two substantially different degeneration processes arising in the algebraic Lie theory.

The first is the study of toric degenerations of flag varieties $G / B$ of complex semisimple Lie algebras, the totality of which is encoded in tropical flag varieties with respect to Plücker embeddings. The second is the PBW-type degenerations of quantized enveloping algebras $U_{q}(\mathfrak{g})$ associated with complex semisimple Lie algebras.

Our link between these two processes is provided by a class of polyhedral cones $\mathcal{D}_{\underline{w}_{0}}^{q}(\mathfrak{g})$, called quantum degree cones [2,9], depending on the complex simple Lie algebra $\mathfrak{g}$ and a choice of $\underline{w}_{0}$, a reduced decomposition of the longest element in the Weyl group of $\mathfrak{g}$. For such a fixed reduced decomposition, the negative part $U_{q}\left(\mathfrak{n}^{-}\right)$of the quantized enveloping algebra is generated by the quantum PBW root vectors with respect to some non-commutative straightening relations. The quantum degree cone is a kind of Gröbner fan in this non-commutative setup, where the monomial ordering is

\footnotetext{
$\varangle \quad$ Xin Fang

xinfang.math@gmail.com

Ghislain Fourier

fourier@art.rwth-aachen.de

Markus Reineke

markus.reineke@rub.de

1 University of Cologne, Mathematical Institute, Weyertal 86-90, 50931 Cologne, Germany

2 RWTH Aachen University, Pontdriesch 10-16, 52062 Aachen, Germany

3 Ruhr-Universität Bochum, Faculty of Mathematics, Universitätsstraße 150, 44780 Bochum, Germany
} 
encoded in $\underline{w}_{0}$. Interior points of such cones give non-commutative degenerations of $U_{q}\left(\mathfrak{n}^{-}\right)$to skew polynomial algebras. As in the commutative Gröbner theory, due to lacking of knowledge on the straightening relations, the facets of the quantum degree cones are usually hard to describe.

The first main result of the present work (Corollary 1) is an embedding (conjectured to be an equality, see Conjecture 1) of the quantum degree cone into the negative tight monomial cone of the Langlands dual Lie algebra, which is a variant of a cone introduced by Lusztig [13] for the identification of monomial elements in the canonical basis of $U_{q}\left(\mathfrak{n}^{-}\right)$. The proof of this result is based on a detailed analysis of a result of Levendorskii and Soibelman [11] on commutators of quantum PBW root vectors, for which we identify a certain PBW basis element appearing in this commutator with nonvanishing coefficient.

The second main result (Theorem 4) is a proof of the aforementioned conjecture for reduced decompositions adapted to a Dynkin quiver. For the proof, we interpret the quantum degree cone $\mathcal{D}_{\underline{w}_{0}}^{q}(\mathfrak{g})$ in terms of the $K$-theory of quiver representations, and use known results on their Auslander-Reiten theory [1], which translate to the quantum group setup via Hall algebras [15].

Tropical flag varieties [4] are subfans of Gröbner fans of flag varieties with respect to Plücker embeddings. We observe that, via a result in [7], the quantum degree cone arising from type $\mathrm{A}$ and a specific choice of reduced decomposition can be identified with a maximal prime cone in the corresponding tropical flag variety (Theorem 5). This observation is quite surprising, as both cones arise from apparently different Gröbner theory: The straightening relations in the quantum setup become trivial after specialization!

The authors hope that the present observation can be further developed into a more general link allowing the study of tropical flag varieties using the piece-wise linear combinatorics arising in quantized enveloping algebras.

The paper is organized as follows: In Sect. 2, we first recall the construction of PBW bases in quantized enveloping algebras and state the commutation formula for root elements. Most of this section is devoted to the proof of the nonvanishing result (Theorem 3) stated above. This result allows us to relate the quantum degree cone and the negative tight monomial cone in Sect. 3, and to formulate our main conjecture (Conjecture 1). In Sect. 4, we review basic results on the representation theory of Dynkin quivers, and on the relation of their associated Hall algebras to the quantized enveloping algebra $U_{q}\left(\mathfrak{n}^{-}\right)$. This allows us to give a $K$-theoretic interpretation of the quantum degree cone and to prove our conjecture (Theorem 4) in many cases. Finally, in Sect. 5 we rephrase the main result of [7] in the present language, identifying a maximal prime cone in the tropical flag variety with a particular quantum degree cone. 


\section{Commutation relations of quantum PBW root vectors}

\subsection{Basics on Lie algebras and quantum groups}

Let $\mathfrak{g}$ be a simple Lie algebra of rank $n$, with Cartan matrix $A=\left(a_{i j}\right)_{n \times n}$. We fix $\Delta_{+}$ to be the set of positive roots and $\alpha_{1}, \ldots, \alpha_{n}$ to be the simple roots in $\Delta_{+}$. The root lattice will be denoted by $\mathcal{Q}$ and the weight lattice by $\mathcal{P}$. We set $\mathcal{Q}_{+}:=\mathbb{N} \Delta_{+}$. Let $W$ be the Weyl group of $\mathfrak{g}$ generated by the simple reflections $s_{1}, \cdots, s_{n}$ and $(-,-)$ the $W$-invariant scalar product on the weight lattice such that: For any short root $\alpha$, $(\alpha, \alpha)=2$; when $\mathfrak{g}=\mathrm{B}_{\mathrm{n}}, \mathrm{C}_{\mathrm{n}}, \mathrm{F}_{4}$, for any long root $\beta,(\beta, \beta)=4$; for $\mathfrak{g}=\mathrm{G}_{2}$ and the long root $\gamma,(\gamma, \gamma)=6$.

For a positive root $\alpha \in \Delta_{+}$, we denote $d_{\alpha}:=(\alpha, \alpha) / 2$, and for any simple root $\alpha_{i}$, $d_{i}:=d_{\alpha_{i}}$. Let $\alpha^{\vee}=d_{\alpha}^{-1} \alpha=2 \alpha /(\alpha, \alpha)$. For $\lambda \in \mathcal{P}$ and $\alpha \in \mathcal{Q},\left\langle\lambda, \alpha^{\vee}\right\rangle:=\frac{2(\lambda, \alpha)}{(\alpha, \alpha)}$. Then the entries of the Cartan matrix are given by: $a_{i j}=\left\langle\alpha_{j}, \alpha_{i}^{\vee}\right\rangle$.

The simple reflections are defined by: $s_{i}(\mu)=\mu-\left\langle\mu, \alpha_{i}^{\vee}\right\rangle \alpha_{i}$. In particular, $s_{i}\left(\alpha_{j}\right)=\alpha_{j}-a_{i j} \alpha_{i}$.

We fix several Lie theoretic notations. Let $w_{0} \in W$ be the longest element in the Weyl group and $R\left(w_{0}\right)$ the set of all reduced decompositions of $w_{0}$. For a reduced expression $\underline{w}_{0}=s_{i_{1}} \cdots s_{i_{N}}$ where $N=\# \Delta_{+}$, we denote $\beta_{t}=s_{i_{1}} \cdots s_{i_{t-1}}\left(\alpha_{i_{t}}\right)$ for $1 \leq t \leq N$; then, $\Delta_{+}=\left\{\beta_{t} \mid t=1, \cdots, N\right\}$.

Let $U_{q}(\mathfrak{g})$ be the generic quantum group associated with $\mathfrak{g}$. It is generated as an algebra by $E_{i}, F_{i}$ and $K_{i}^{ \pm 1}$ for $i=1, \cdots, n$, subject to the following relations: For $i, j=1, \cdots, n$,

$$
\begin{aligned}
K_{i} K_{i}^{-1} & =K_{i}^{-1} K_{i}=1, \quad K_{i} E_{j} K_{i}^{-1}=q_{i}^{a_{i j}} E_{j}, \quad K_{i} F_{j} K_{i}^{-1}=q_{i}^{-a_{i j}} F_{j}, \\
E_{i} F_{j} & -F_{j} E_{i}=\delta_{i j} \frac{K_{i}-K_{i}^{-1}}{q_{i}-q_{i}^{-1}}
\end{aligned}
$$

and for $i \neq j$,

$$
\sum_{r=0}^{1-a_{i j}}(-1)^{r} E_{i}^{\left(1-a_{i j}-r\right)} E_{j} E_{i}^{(r)}=0, \quad \sum_{r=0}^{1-a_{i j}}(-1)^{r} F_{i}^{\left(1-a_{i j}-r\right)} F_{j} F_{i}^{(r)}=0,
$$

where

$$
q_{i}=q^{d_{i}}, \quad[n]_{q} !=\prod_{i=1}^{n} \frac{q^{n}-q^{-n}}{q-q^{-1}}, \quad E_{i}^{(n)}=\frac{E_{i}^{n}}{[n]_{q_{i}} !} \text { and } F_{i}^{(n)}=\frac{F_{i}^{n}}{[n]_{q_{i}} !}
$$

Let $U_{q}\left(\mathfrak{n}^{-}\right)$be the subalgebra of $U_{q}(\mathfrak{g})$ generated by $F_{i}$ for $i=1, \cdots, n$.

Let $T_{i}=T_{i, 1}^{\prime \prime}, i=1, \cdots, n$ be Lusztig's automorphisms:

$$
T_{i}\left(E_{i}\right)=-F_{i} K_{i}, \quad T_{i}\left(F_{i}\right)=-K_{i}^{-1} E_{i}, \quad T_{i}\left(K_{j}\right)=K_{j} K_{i}^{-a_{i j}},
$$


for $i=1, \cdots, n$, and for $j \neq i$,

$$
T_{i}\left(E_{j}\right)=\sum_{r+s=-a_{i j}}(-1)^{r} q_{i}^{-r} E_{i}^{(s)} E_{j} E_{i}^{(r)}, \quad T_{i}\left(F_{j}\right)=\sum_{r+s=-a_{i j}}(-1)^{r} q_{i}^{r} F_{i}^{(r)} F_{j} F_{i}^{(s)} .
$$

Details can be found in Chapter 37 of [12]. The quantum PBW root vector $F_{\beta_{t}}$ associated with $\beta_{t}$ is defined by:

$$
F_{\beta_{t}}:=T_{i_{1}} T_{i_{2}} \cdots T_{i_{t-1}}\left(F_{i_{t}}\right) \in U_{q}\left(\mathfrak{n}^{-}\right)
$$

Note that for $i=1, \cdots, n, F_{\alpha_{i}}=F_{i}$. For $s \in \mathbb{N}$, we define

$$
F_{\beta_{t}}^{(s)}:=\frac{F_{\beta_{t}}^{s}}{[s]_{q_{t}} !}
$$

The PBW theorem of quantum groups says that the set

$$
\left\{F_{\beta_{1}}^{\left(c_{1}\right)} F_{\beta_{2}}^{\left(c_{2}\right)} \cdots F_{\beta_{N}}^{\left(c_{N}\right)} \mid\left(c_{1}, \cdots, c_{N}\right) \in \mathbb{N}^{N}\right\}
$$

is a linear basis of $U_{q}\left(\mathfrak{n}^{-}\right)$([12], Corollary 40.2.2).

\subsection{Commutation relations of quantum PBW root vectors}

The commutation relations of these quantum PBW root vectors are described in the following Levendorskii-Soibelman (LS for short) formula:

Theorem 1 ([6,11]). For any $1 \leq k<\ell \leq N$,

$$
F_{\beta_{\ell}} F_{\beta_{k}}-q^{-\left(\beta_{k}, \beta_{\ell}\right)} F_{\beta_{k}} F_{\beta_{\ell}}=\sum_{n_{k+1}, \ldots, n_{\ell-1} \geq 0} c\left(n_{k+1}, \ldots, n_{\ell-1}\right) F_{\beta_{k+1}}^{\left(n_{k+1}\right)} \ldots F_{\beta_{\ell-1}}^{\left(n_{\ell-1}\right)},
$$

where $c\left(n_{k+1}, \ldots, n_{\ell-1}\right) \in \mathbb{Z}\left[q^{ \pm 1}\right]$.

A more general result is proved by $\mathrm{Xi}$ in [17]:

Theorem 2 ([17]). For any $1 \leq k<\ell \leq N$ and $s, t \in \mathbb{N}$,

$$
F_{\beta_{\ell}}^{(s)} F_{\beta_{k}}^{(t)}-q^{-s t\left(\beta_{k}, \beta_{\ell}\right)} F_{\beta_{k}}^{(t)} F_{\beta_{\ell}}^{(s)}=\sum_{n_{k}, \ldots, n_{\ell} \geq 0, n_{k}<t, n_{\ell}<s} c\left(n_{k}, \ldots, n_{\ell}\right) F_{\beta_{k}}^{\left(n_{k}\right)} \ldots F_{\beta_{\ell}}^{\left(n_{\ell}\right)},
$$

where $c\left(n_{k}, \ldots, n_{\ell}\right) \in \mathbb{Z}\left[q^{ \pm 1}\right]$.

Since the total order $\beta_{1}<\beta_{2}<\ldots<\beta_{N}$ is convex, by specializing $q$ to 1 , we know that in the LS formula (2.1), if there exists $k<r<\ell$ such that $\beta_{r}=\beta_{k}+\beta_{\ell}$, then the monomial $F_{\beta_{r}}$ appears on the right-hand side of (2.1) with nonzero coefficient. 
These are all information that can be read off from the specialization $q \rightarrow 1$. It is natural to ask: What are the monomials appearing on the right-hand side of (2.1) with nonzero coefficients?

The goal of the rest of this section is to show that particular monomials may appear in some situations. This will be applied later to study the quantum degree cones.

For $1 \leq k \leq N$ and $s \in \mathbb{N}$, we define $k[s]:=t$ if $t>k$ such that $i_{t}=i_{k}$ and the number of $i_{k}$ appearing in $\left\{i_{k+1}, \ldots, i_{t}\right\}$ is exactly $s$; otherwise, we set it to be zero. Hence, $k[1]=\ell$ implies that $i_{k}=i_{\ell}$ and for any $k<p<\ell, i_{p} \neq i_{k}$.

Theorem 3 Suppose that $k[1]=\ell$ and $c_{s}=-\left\langle\alpha_{i_{k}}, \alpha_{i_{s}}^{\vee}\right\rangle$. The monomial $F_{\beta_{k+1}}^{\left(c_{k+1}\right)} \ldots F_{\beta_{\ell-1}}^{\left(c_{\ell-1}\right)}$ appears in $F_{\beta_{\ell}} F_{\beta_{k}}-q^{-\left(\beta_{k}, \beta_{\ell}\right)} F_{\beta_{k}} F_{\beta_{\ell}}$ with nonzero coefficient.

The rest of this section will be devoted to proving this theorem.

First observe that by applying $T_{i_{k-1}}^{-1} \ldots T_{i_{1}}^{-1}$, we can suppose that $k=1$. By assumption, $i_{\ell}=i_{1}$.

\subsection{Preparations to the proof}

We keep the notations in the statement of the theorem and start deriving some combinatorial formulas on roots.

Lemma 1 The following statements hold:

(1). We have $\beta_{1}+\beta_{\ell}=\sum_{j=2}^{\ell-1} c_{j} \beta_{j}$.

(2). When $\ell>3$ and $c_{2} \neq 0$, the root $s_{i_{1}} \ldots s_{i_{\ell-2}}\left(\alpha_{i_{1}}\right) \in \mathcal{Q}_{+}$.

Proof (1) By the definition of $\beta_{\ell}$, we have:

$$
\begin{aligned}
\beta_{\ell} & =s_{i_{1}} \ldots s_{i_{\ell-2}} s_{i_{\ell-1}}\left(\alpha_{i_{1}}\right) \\
& =s_{i_{1}} \ldots s_{i_{\ell-2}}\left(\alpha_{i_{1}}+c_{\ell-1} \alpha_{i_{\ell-1}}\right) \\
& =s_{i_{1}} \ldots s_{i_{\ell-2}}\left(\alpha_{i_{1}}\right)+c_{\ell-1} \beta_{\ell-1} .
\end{aligned}
$$

Iterating this computation gives

$$
\beta_{\ell}=s_{i_{1}}\left(\alpha_{i_{1}}\right)+c_{2} \beta_{2}+\ldots+c_{\ell-1} \beta_{\ell-1} .
$$

As $\beta_{1}=\alpha_{i_{1}}$, the formula is proved.

(2) The same argument as in (1) shows that

$$
s_{i_{1}} \ldots s_{i_{\ell-2}}\left(\alpha_{i_{1}}\right)=-\alpha_{i_{1}}+c_{2} \beta_{2}+\ldots+c_{\ell-2} \beta_{\ell-2}
$$

Since for any $2 \leq s \leq \ell-1, c_{s} \geq 0$, it suffices to show that $c_{2} \beta_{2}-\alpha_{i_{1}}$ is a positive root. We have

$$
c_{2} \beta_{2}-\alpha_{i_{1}}=c_{2} \alpha_{i_{2}}-\left(c_{2} a_{i_{1}, i_{2}}+1\right) \alpha_{i_{1}} .
$$

As $c_{2}=-\left\langle\alpha_{i_{1}}, \alpha_{i_{2}}^{\vee}\right\rangle \geq 1, a_{i_{1}, i_{2}}=\left\langle\alpha_{i_{2}}, \alpha_{i_{1}}^{\vee}\right\rangle \leq-1$, this proves the statement. 
The following lemma will be used later.

Lemma 2 Assume that $\ell>3, c_{2} \neq 0$ and let $X:=T_{i_{1}} \ldots T_{i_{\ell-2}}\left(F_{i_{1}}\right)$.

(1) We have $X \in U_{q}\left(\mathfrak{n}^{-}\right)$.

(2) In the following expression of $X$ :

$$
X=\sum_{n_{1}, \ldots, n_{N} \geq 0} h\left(n_{1}, \ldots, n_{N}\right) F_{\beta_{1}}^{\left(n_{1}\right)} \ldots F_{\beta_{N}}^{\left(n_{N}\right)},
$$

if $h\left(n_{1}, \ldots, n_{N}\right) \neq 0$, then $n_{\ell-1}=n_{\ell}=\ldots=n_{N}=0$.

Proof (1) The quantum group $U_{q}(\mathfrak{g})$ is graded by the root lattice $\mathcal{Q}$ where $E_{i}$ (resp. $F_{i}$ ) has degree $\alpha_{i}$ (resp. $-\alpha_{i}$ ) and $K_{i}^{ \pm 1}$ have degree 0 . The Lusztig automorphism $T_{i}$ preserves this grading and sends a homogeneous element of degree $\beta \in \mathcal{Q}$ to a homogeneous element of degree $s_{i}(\beta)$. According to Lemma 1 (3), the statement holds since $X$ is homogeneous of degree $-s_{i_{1}} \cdots s_{i_{\ell-2}}\left(\alpha_{i_{1}}\right)$.

(2) Since $c_{2} \neq 0$, there exists $2 \leq t \leq \ell-2$ such that $c_{t} \neq 0$ and for any $t<p \leq \ell-2$, $c_{p}=0$. Then

$$
\begin{aligned}
X & =T_{i_{1}} \ldots T_{i_{t}}\left(F_{i_{1}}\right) \\
& =\sum_{r+s=c_{t}}(-1)^{r} q_{i_{t}}^{r} T_{i_{1}} \ldots T_{i_{t-1}}\left(F_{i_{t}}^{(r)}\right) T_{i_{1}} \ldots T_{i_{t-1}}\left(F_{i_{1}}\right) T_{i_{1}} \ldots T_{i_{t-1}}\left(F_{i_{t}}^{(s)}\right) \\
& =\sum_{r+s=c_{t}}(-1)^{r} q_{i_{t}}^{r} F_{\beta_{t}}^{(r)} T_{i_{1}} \ldots T_{i_{t-1}}\left(F_{i_{1}}\right) F_{\beta_{t}}^{(s)} .
\end{aligned}
$$

Since $t-1<\ell-2$, iterating this procedure and applying Theorem 2 to write the quantum PBW root vectors into correct order, we obtained that $n_{t+1}=n_{t+2}=$ $\ldots=n_{N}=0$. As $t \leq \ell-2, n_{\ell-1}=n_{\ell}=\ldots=n_{N}=0$.

\subsection{Proof of Theorem 3: rank 2 cases}

When the rank of $\mathfrak{g}$ is 2 , to prove the theorem, it suffices to consider the cases $\left(i_{1}, i_{2}, i_{3}\right)=(1,2,1)$ and $\left(i_{1}, i_{2}, i_{3}\right)=(2,1,2)$.

We first prove the theorem for $\left(i_{1}, i_{2}, i_{3}\right)=(2,1,2)$ in $A_{2}, B_{2}$ and $C_{2}$, and the other case can be proved similarly.

(1) Case $A_{2}$. In this case, $\beta_{1}=\alpha_{2}, \beta_{3}=\alpha_{1}$; hence, $F_{\beta_{3}}=F_{1}$. The commutation relation reads $F_{1} F_{2}-q F_{2} F_{1}$, which is nothing but $T_{2}\left(F_{1}\right)$.

(2) Case $\mathrm{B}_{2}$. In this case, $\beta_{1}=\alpha_{2}, \beta_{3}=2 \alpha_{1}+\alpha_{2}$. The commutation relation is

$$
F_{\beta_{3}} F_{\beta_{1}}-F_{\beta_{1}} F_{\beta_{3}}=\left(-1+q^{-2}\right) F_{\beta_{2}}^{(2)} .
$$

(3) Case $C_{2}$. In this case, $\beta_{1}=\alpha_{2}, \beta_{3}=\alpha_{1}+\alpha_{2}$. The commutation relation is given by

$$
F_{\beta_{3}} F_{\beta_{1}}-F_{\beta_{1}} F_{\beta_{3}}=\left(q+q^{-1}\right) F_{\beta_{2}}
$$


It remains to consider the $\mathrm{G}_{2}$ case. For $\left(i_{1}, i_{2}, i_{3}\right)=(1,2,1), \beta_{1}=\alpha_{1}$ and $\beta_{3}=$ $2 \alpha_{1}+3 \alpha_{2}$. The commutation relation reads

$$
F_{\beta_{3}} F_{\beta_{1}}-q^{-3} F_{\beta_{1}} F_{\beta_{3}}=\left(1-q^{-2}-q^{-4}+q^{-6}\right) F_{\beta_{2}}^{(3)} .
$$

For $\left(i_{1}, i_{2}, i_{3}\right)=(2,1,2), \beta_{1}=\alpha_{2}$ and $\beta_{3}=\alpha_{1}+2 \alpha_{2}$. The commutation relation is

$$
F_{\beta_{3}} F_{\beta_{1}}-q^{-1} F_{\beta_{1}} F_{\beta_{3}}=\left(q^{2}+1+q^{-2}\right) F_{\beta_{2}} .
$$

\subsection{Proof of Theorem 3: general case}

The proof is executed by induction on $\ell$. The case $\ell=3$ is the rank 2 case proved above.

Moreover, we can assume that $c_{2} \neq 0$ and $c_{\ell-1} \neq 0$, since otherwise the statement holds by induction hypothesis.

We start with considering the quantum PBW root vector $F_{\beta_{\ell}}$ :

$$
\begin{aligned}
F_{\beta_{\ell}} & =T_{i_{1}} \ldots T_{i_{\ell-1}}\left(F_{i_{1}}\right) \\
& =\sum_{r+s=c_{\ell-1}}(-1)^{r} q_{i_{\ell-1}}^{r} T_{i_{1}} \ldots T_{i_{\ell-2}}\left(F_{i_{\ell-1}}^{(r)} F_{i_{1}} F_{i_{\ell-1}}^{(s)}\right) \\
& =\sum_{r+s=c_{\ell-1}}(-1)^{r} q_{i_{\ell-1}}^{r} F_{\beta_{\ell-1}}^{(r)} T_{i_{1}} \ldots T_{i_{\ell-2}}\left(F_{i_{1}}\right) F_{\beta_{\ell-1}}^{(s)} .
\end{aligned}
$$

We suppose that $X=T_{i_{1}} \ldots T_{i_{\ell-2}}\left(F_{i_{1}}\right)$ and $\gamma=s_{i_{1}} \ldots s_{i_{\ell-2}}\left(\alpha_{i_{1}}\right)$. The commutator of quantum $\mathrm{PBW}$ root vectors gives

$$
F_{\beta_{\ell}} F_{\beta_{1}}-q^{-\left(\beta_{1}, \beta_{\ell}\right)} F_{\beta_{1}} F_{\beta_{\ell}}=\sum_{r+s=c_{\ell-1}}(-1)^{r} q_{i_{\ell-1}}^{r}\left(F_{\beta_{\ell-1}}^{(r)} X F_{\beta_{\ell-1}}^{(s)} F_{\beta_{1}}-q^{-\left(\beta_{1}, \beta_{\ell}\right)} F_{\beta_{1}} F_{\beta_{\ell-1}}^{(r)} X F_{\beta_{\ell-1}}^{(s)}\right) .
$$

From Theorem 2, we can write

$$
F_{\beta_{\ell-1}}^{(s)} F_{\beta_{1}}=q^{-s\left(\beta_{1}, \beta_{\ell-1}\right)} F_{\beta_{1}} F_{\beta_{\ell-1}}^{(s)}+\Sigma_{1}^{\prime},
$$

where $\Sigma_{1}^{\prime}$ consists of terms appearing on the right-hand side of (2.2); moreover, we set $\Sigma_{1}:=F_{\beta_{\ell-1}}^{(r)} X \Sigma_{1}^{\prime}$; then,

$$
F_{\beta_{\ell-1}}^{(r)} X F_{\beta_{\ell-1}}^{(s)} F_{\beta_{1}}=q^{-s\left(\beta_{1}, \beta_{\ell-1}\right)} F_{\beta_{\ell-1}}^{(r)} X F_{\beta_{1}} F_{\beta_{\ell-1}}^{(s)}+\Sigma_{1}
$$

We claim that $F_{\beta_{2}}^{\left(c_{2}\right)} \ldots F_{\beta_{\ell-1}}^{\left(c_{\ell-1}\right)}$ does not appear with nonzero coefficient in $\Sigma_{1}$. Indeed, by Lemma 2, writing $X$ in the PBW basis, $F_{\beta_{\ell-1}}$ does not appear in any monomial. Hence, commuting $F_{\beta_{\ell-1}}^{(r)}$ with $X$ using Theorem 2 will not producing any element in the PBW basis with the power of $F_{\beta_{\ell-1}}$ higher than $r$. Again by Theorem 2, commuting $F_{\beta_{\ell-1}}^{(s)}$ and $F_{\beta_{1}}$ will produce elements in the PBW basis with the power of $F_{\beta_{\ell-1}}$ strictly less than $s$. Combining them together proves the claim. 
The same argument shows that, if we write using Theorem 2

$$
F_{\beta_{1}} F_{\beta_{\ell-1}}^{(r)} X F_{\beta_{\ell-1}}^{(s)}=q^{-r\left(\beta_{1}, \beta_{\ell-1}\right)} F_{\beta_{\ell-1}}^{(r)} F_{\beta_{1}} X F_{\beta_{\ell-1}}^{(s)}+\Sigma_{2}
$$

where $\Sigma_{2}$ is defined in the same way as $\Sigma_{1}$, then the monomial $F_{\beta_{2}}^{\left(c_{2}\right)} \ldots F_{\beta_{\ell-1}}^{\left(c_{\ell-1}\right)}$ does not appear in $\Sigma_{2}$ with nonzero coefficient.

As a consequence, to study the coefficient of $F_{\beta_{2}}^{\left(c_{2}\right)} \ldots F_{\beta_{\ell-1}}^{\left(c_{\ell-1}\right)}$, it suffices to consider the term

$$
\begin{aligned}
& \sum_{r+s=c_{\ell-1}}(-1)^{r} q_{i_{\ell-1}}^{r}\left(q^{-s\left(\beta_{1}, \beta_{\ell-1}\right)} F_{\beta_{\ell-1}}^{(r)} X F_{\beta_{1}} F_{\beta_{\ell-1}}^{(s)}-q^{r\left(\beta_{1}, \beta_{\ell-1}\right)-\left(\beta_{1}, \beta_{\ell}\right)} F_{\beta_{\ell-1}}^{(r)} F_{\beta_{1}} X F_{\beta_{\ell-1}}^{(s)}\right) \\
& =\sum_{r+s=c_{\ell-1}}(-1)^{r} q_{i_{\ell-1}}^{r} q^{-s\left(\beta_{1}, \beta_{\ell-1}\right)} F_{\beta_{\ell-1}}^{(r)}\left(X F_{\beta_{1}}-q^{c_{\ell-1}\left(\beta_{1}, \beta_{\ell-1}\right)-\left(\beta_{1}, \beta_{\ell}\right)} F_{\beta_{1}} X\right) F_{\beta_{\ell-1}}^{(s)}
\end{aligned}
$$

We simplify the power of $q$ in the brackets: By the proof of Lemma 1 (1),

$$
c_{\ell-1}\left(\beta_{1}, \beta_{\ell-1}\right)-\left(\beta_{1}, \beta_{\ell}\right)=-\left(\beta_{1}, \beta_{\ell}-c_{\ell-1} \beta_{\ell-1}\right)=-\left(\beta_{1}, \gamma\right) .
$$

The term in the brackets now reads

$$
T_{i_{1}} \ldots T_{i_{\ell-2}}\left(F_{i_{1}}\right) F_{\beta_{1}}-q^{-\left(\beta_{1}, \gamma\right)} F_{\beta_{1}} T_{i_{1}} \ldots T_{i_{\ell-2}}\left(F_{i_{1}}\right) .
$$

By induction hypothesis, in this commutator, when written in the PBW basis, there exists a term $\lambda F_{\beta_{2}}^{\left(c_{2}\right)} \ldots F_{\beta_{\ell-2}}^{\left(c_{\ell-2}\right)}$ with $\lambda \neq 0$. From this, we obtain the following term in the above sum

$$
\sum_{r+s=c_{\ell-1}}(-1)^{r} q_{i_{\ell-1}}^{r} q^{-s\left(\beta_{1}, \beta_{\ell-1}\right)} \lambda F_{\beta_{\ell-1}}^{(r)} F_{\beta_{2}}^{\left(c_{2}\right)} \ldots F_{\beta_{\ell-2}}^{\left(c_{\ell-2}\right)} F_{\beta_{\ell-1}}^{(s)} .
$$

We denote

$$
\Sigma_{3}:=F_{\beta_{\ell-1}}^{(r)} F_{\beta_{2}}^{\left(c_{2}\right)} \ldots F_{\beta_{\ell-2}}^{\left(c_{\ell-2}\right)}-q^{-\mu} F_{\beta_{2}}^{\left(c_{2}\right)} \ldots F_{\beta_{\ell-2}}^{\left(c_{\ell-2}\right)} F_{\beta_{\ell-1}}^{(r)}
$$

where $\mu=\sum_{t=2}^{\ell-2} c_{t} r\left(\beta_{t}, \beta_{\ell-1}\right)$. By Theorem 2 again, the monomial $F_{\beta_{2}}^{\left(c_{2}\right)} \ldots$ $F_{\beta_{\ell-2}}^{\left(c_{\ell-2}\right)} F_{\beta_{\ell-1}}^{(r)}$ does not appear in $\Sigma_{3}$ with nonzero coefficient. Therefore, the monomial $F_{\beta_{2}}^{\left(c_{2}\right)} \ldots F_{\beta_{\ell-2}}^{\left(c_{\ell-2}\right)} F_{\beta_{\ell-1}}^{\left(c_{\ell-1}\right)}$ appears in (2.3) with coefficient

$$
\lambda \sum_{r+s=c_{\ell-1}}(-1)^{r} q_{i_{\ell-1}}^{r} q^{-s\left(\beta_{1}, \beta_{\ell-1}\right)-\sum_{t=2}^{\ell-2} c_{t} r\left(\beta_{t}, \beta_{\ell-1}\right)}\left[\begin{array}{c}
c_{\ell-1} \\
q
\end{array}\right]_{q_{i_{\ell-1}}}
$$

It suffices to show that this term is nonzero. We first simplify the power of $q$ : apply Lemma 1 (1) and replace $s=c_{\ell-1}-r$,

$$
s\left(\beta_{1}, \beta_{\ell-1}\right)+\sum_{t=2}^{\ell-2} c_{t} r\left(\beta_{t}, \beta_{\ell-1}\right)=c_{\ell-1}\left(\beta_{1}, \beta_{\ell-1}\right)+r\left(-\beta_{1}+\sum_{t=2}^{\ell-2} c_{t} \beta_{t}, \beta_{\ell-1}\right)
$$




$$
\begin{aligned}
& =c_{\ell-1}\left(\beta_{1}, \beta_{\ell-1}\right)+r\left(\beta_{\ell}-c_{\ell-1} \beta_{\ell-1}, \beta_{\ell-1}\right) \\
& =c_{\ell-1}\left(\beta_{1}, \beta_{\ell-1}\right)+r\left(s_{i_{\ell-1}}\left(\alpha_{i_{1}}\right)-c_{\ell-1} \alpha_{i_{\ell-1}}, \alpha_{i_{\ell-1}}\right) \\
& =c_{\ell-1}\left(\beta_{1}, \beta_{\ell-1}\right)+r\left(\alpha_{i_{1}}, \alpha_{i_{\ell-1}}\right)
\end{aligned}
$$

where for the third equality, the $W$-invariance is used. Since both $\lambda$ and $q^{-\left(\beta_{1}, \beta_{\ell-1}\right)}$ are nonzero constants, to show that the coefficient (2.5) is nonzero, it suffices to show that

$$
\sum_{r+s=c_{\ell-1}}(-1)^{r} q_{i_{\ell-1}}^{r} q^{-r\left(\alpha_{i_{1}}, \alpha_{i_{\ell-1}}\right)}\left[\begin{array}{c}
c_{\ell-1} \\
q
\end{array}\right]_{q_{i_{\ell-1}}}=\sum_{r+s=c_{\ell-1}}(-1)^{r} q_{i_{\ell-1}}^{r\left(1+c_{\ell-1}\right)}\left[\begin{array}{c}
c_{\ell-1} \\
q
\end{array}\right]_{q_{i_{\ell-1}}} \neq 0 .
$$

Here, we used the fact that $c_{\ell-1}=-d_{i_{\ell-1}}^{-1}\left(\alpha_{i_{\ell-1}}, \alpha_{i_{1}}\right)$.

By the assumption, $c_{\ell-1} \neq 0$; hence, in the case where the rank of $\mathfrak{g}$ is higher than $2, c_{\ell-1}=1$ or 2 . If $c_{\ell-1}=1$, equation (2.6) reads $1-q_{i_{\ell-1}}^{2}$. If $c_{\ell-1}=2$, equation (2.6) reads $1-q_{i_{\ell-1}}^{2}-q_{i_{\ell-1}}^{4}+q_{i_{\ell-1}}^{6}$. In both cases, they are nonzero. This terminates the proof of Theorem 3 .

Remark 1 It is clear from the proof that Theorem 3 still holds when $q$ is not a small root of unity.

\section{Quantum degree cone and Lusztig tight monomial cone}

\subsection{Lusztig tight monomial cones}

In [13], Lusztig defined a polyhedral cone in the simply laced cases in order to study monomials in the canonical basis. The natural generalizations to all Lie types are given in [5].

Let $\underline{w}_{0}=s_{i_{1}} \cdots s_{i_{N}}$ be a reduced decomposition of $w_{0}$. The Lusztig tight monomial cone $\mathcal{L}_{\underline{w}_{0}}(\mathfrak{g}) \subset \mathbb{R}^{N}$ is the polyhedral cone defined by the following inequalities:

(i) for any two indices $1 \leq p<p^{\prime} \leq N$ with $p^{\prime}=p[1]$, we have

$$
x_{p}+x_{p^{\prime}}+\sum_{p<s<p^{\prime}} a_{i_{p}, i_{s}} x_{s} \leq 0
$$

(ii) for $1 \leq p \leq N, x_{p} \geq 0$.

It is shown in [5, Theorem 8.10] that the cone $\mathcal{L}_{\underline{w}_{0}}(\mathfrak{g})$ is simplicial.

We will denote $\mathcal{L}_{\underline{w}_{0}}^{-}(\mathfrak{g}) \subset \mathbb{R}^{N}$, termed a negative tight monomial cone, the polyhedral cone defined by

(i)' for any two indices $1 \leq p<p^{\prime} \leq N$ with $p^{\prime}=p[1]$, we have

$$
x_{p}+x_{p^{\prime}}+\sum_{p<s<p^{\prime}} a_{i_{p}, i_{s}} x_{s} \geq 0 .
$$


The cone $\mathcal{L}_{\underline{w}_{0}}^{-}(\mathfrak{g})$ is a product of a lineality space of dimension $n$ and a simplicial cone of dimension $N-n$. Indeed, the statement on lineality space holds since the matrix defining the polyhedral cone has rank $N-n$. The simplicial property then follows as there are exactly $N-n$ inequalities defining $\mathcal{L}_{\underline{w}_{0}}^{-}(\mathfrak{g})$.

\subsection{Quantum degree cones}

The quantum degree cones are defined in [2], motivated by studying the quantum PBW filtration on quantum groups.

We keep the notations in LS formula. The quantum degree cone $\mathcal{D}_{\underline{w}_{0}}^{q}(\mathfrak{g})$ associated with a reduced decomposition $\underline{w}_{0}$ is defined by:

$$
\begin{aligned}
& \mathcal{D}_{\underline{w}_{0}}^{q}(\mathfrak{g}):=\left\{\mathbf{d}=\left(d_{\beta}\right)_{\beta \in \Delta_{+}} \in \mathbb{R}^{\Delta_{+}} \mid \text {for } i<j,\right. \\
& \left.d_{\beta_{i}}+d_{\beta_{j}} \geq \sum_{k=i+1}^{j-1} n_{k} d_{\beta_{k}} \text { if } c\left(n_{i+1}, \cdots, n_{j-1}\right) \neq 0\right\} .
\end{aligned}
$$

Our notation here is slightly different to [2]: The quantum degree cone therein is the interior of the cone $\mathcal{D}_{\underline{w}_{0}}^{q}(\mathfrak{g}) \cap \mathbb{R}_{\geq 0}^{\Delta_{+}}$.

It is proved in loc.cit. that $\mathcal{D}_{\underline{w}_{0}}^{q}(\mathfrak{g})$ is non-empty, and any $\mathbf{d} \in \mathcal{D}_{\underline{w}_{0}}^{q}(\mathfrak{g})$ defines a filtered algebra structure on $U_{q}\left(\overline{\mathfrak{n}}^{-}\right)$by letting $\operatorname{deg}\left(F_{\beta_{i}}\right)=d_{\beta_{i}}$. The associated graded algebra is isomorphic to a skew polynomial algebra if and only if $\mathbf{d}$ is contained in the interior of $\mathcal{D}_{\underline{w}_{0}}^{q}(\mathfrak{g})$.

Remark 2 It is natural to ask for whether it is possible to glue these separate cones into a polyhedral fan. Some related results can be found in [2, Lemma 3.3, Remark 3.4, Theorem 4.5]. For example, in the rank 2 case, it is shown in [2, Proposition 4.4] that all quantum degree cones are the same. We do not have a general answer.

\subsection{Relations between two cones}

For a fixed $\underline{w}_{0}$, we identify $\mathbb{R}^{\Delta_{+}}$with $\mathbb{R}^{N}$ by sending $\left(d_{\beta}\right)_{\beta \in \Delta_{+}}$to $\left(d_{\beta_{1}}, d_{\beta_{2}}, \cdots, d_{\beta_{N}}\right)$.

As a consequence of Theorem 3, for a fixed $\underline{w}_{0}$, we obtain an embedding of the quantum degree cone to the negative tight monomial cone. We denote $\mathfrak{g}^{L}$ the Langlands dual of the Lie algebra $\mathfrak{g}$.

Corollary 1 For any $\underline{w}_{0} \in R\left(w_{0}\right)$, we have $\mathcal{D}_{\underline{w}_{0}}^{q}(\mathfrak{g}) \subseteq \mathcal{L}_{\underline{w}_{0}}^{-}\left(\mathfrak{g}^{L}\right)$.

Proof According to Theorem 3 and the definition of the quantum degree cone, all defining inequalities of $\mathcal{L}_{\underline{w}_{0}}^{-}\left(\mathfrak{g}^{L}\right)$ are fulfilled for any point in the quantum degree cone.

We conjecture that these two cones coincide.

Conjecture 1 For any $\underline{w}_{0} \in R\left(w_{0}\right)$, we have $\mathcal{D}_{\underline{w}_{0}}^{q}(\mathfrak{g})=\mathcal{L}_{\underline{w}_{0}}^{-}\left(\mathfrak{g}^{L}\right)$.

In the next section, we prove this conjecture in the case where $\mathfrak{g}$ is simply laced and $\underline{w}_{0}$ is compatible with a quiver. 


\section{Quiver representations and Hall algebras}

The partial proof of Conjecture 1 makes essential use of the representation theory of Dynkin quivers. Namely, the quantum group $U_{q}\left(\mathfrak{n}^{-}\right)$can be modeled in this language by identifying it with the so-called generic Hall algebra of a Dynkin quiver. In this way, the conjecture can be derived from a statement about $K$-theoretic cones. We will make free use of the basic results on representations of quivers and their Auslander-Reiten theory; a reference is [1]. For Hall algebra methods, we refer to [15], [9].

\subsection{Quiver representations}

Let $\mathbb{K}$ be a field. We fix a Dynkin quiver, that is, an oriented graph whose underlying unoriented graph is a Dynkin diagram of type A, D, or E. We denote the set of vertices of $Q$ by $I$. The number of arrows between any two different vertices $i$ and $j$ (in either direction) thus equals $-a_{i j} \in\{0,1\}$.

Associated with these data, we have the path algebra $\mathbb{K} Q$ of $Q$, which is a finite dimensional associative unital algebra. We denote by $\bmod (\mathbb{K} Q)$ the category of finite dimensional $\mathbb{K} Q$-modules.

We denote by $K_{0}:=K_{0}(\mathbb{K} Q)$ the Grothendieck group of $\mathbb{K} Q$, which is the free abelian group in isomorphism classes $[X]$ in $\bmod (\mathbb{K} Q)$ modulo the relation $[X]=$ $[M]+[N]$ whenever there exists a short exact sequence $0 \rightarrow M \rightarrow X \rightarrow N \rightarrow 0$. By the Jordan-Hoelder theorem, $K_{0}$ is isomorphic to the free abelian group generated by the isomorphism classes of simple modules.

Let $K_{0}^{\oplus}:=K_{0}^{\oplus}(\mathbb{K} Q)$ be the split Grothendieck group of $\mathbb{K} Q$-modules, which is the free abelian group in isomorphism classes in $\bmod (\mathbb{K} Q)$ modulo the relations $[M \oplus N]=[M]+[N]$. By the Krull-Schmidt theorem, $K_{0}^{\oplus}$ is freely generated by the isomorphism classes of indecomposable modules.

By definition, there is a well-defined map dim : $K_{0}^{\oplus} \rightarrow K_{0}$.

The (isomorphism classes of) simple modules $S_{i}$ in $\bmod (\mathbb{K} Q)$ are parametrized by the vertices $i \in I$. This identifies $K_{0} \simeq \mathbb{Z}^{I}$ with the root lattice of $\mathfrak{g}$, the Lie algebra having the underlying graph of $Q$ as Dynkin diagram, by mapping the class $\left[S_{i}\right]$ to the simple root $\alpha_{i}$. By Gabriel's theorem, the (isomorphism classes of) indecomposable modules are parametrized by the positive roots in the root lattice: For each $\alpha \in \Delta_{+}$, there exists a unique (up to isomorphism) indecomposable module $U_{\alpha}$ having dimension vector $\alpha$.

Using these identifications, the map $\operatorname{dim}: K_{0}^{\oplus} \rightarrow K_{0}$ just assigns to an isomorphism class its dimension vector. We can also parametrize all isomorphism classes in $\bmod (\mathbb{K} Q)$ by functions $\varphi: \Delta_{+} \rightarrow \mathbb{N}$ via

$$
M_{\varphi}(\mathbb{K})=\bigoplus_{\alpha \in \Delta_{+}} U_{\alpha}^{\varphi(\alpha)}
$$

Note that this parametrization is independent of the ground field $\mathbb{K}$. 
The category $\bmod (\mathbb{K} Q)$ is of global dimension at most one, that is, $\operatorname{Ext}^{\geq 2}(-,-)$ vanishes identically. For two $\mathbb{K} Q$-modules $M$ and $N$, we will use the shorthand notation

$$
[M, N]:=\operatorname{dim} \operatorname{Hom}(M, N) \text { and }[M, N]^{1}=\operatorname{dim}_{\operatorname{Ext}^{1}}(M, N),
$$

and we define $\langle M, N\rangle=[M, N]-[M, N]^{1}$, the homological Euler form of $Q$. It only depends on the classes of $M$ and $N$ in $K_{0}$; thus, we have a well-defined bilinear form

$$
\langle\operatorname{dim} M, \operatorname{dim} N\rangle=[M, N]-[M, N]^{1}
$$

on the root lattice $\mathcal{Q}$, whose symmetrization $(-,-)$ is known to equal the bilinear form defined by the Cartan matrix.

\subsection{Auslander-Reiten quivers arising from reduced decompositions}

For every non-projective indecomposable $U$ in $\bmod (\mathbb{K} Q)$, there exists a so-called almost split (or Auslander-Reiten) sequence ending in $U$, that is, a non-split short exact sequence

$$
0 \rightarrow \tau U \rightarrow B_{U} \rightarrow U \rightarrow 0
$$

with the following defining property: for every indecomposable $V$, the space $C_{U, V}$ defined by

$$
0 \rightarrow \operatorname{Hom}(V, \tau U) \rightarrow \operatorname{Hom}\left(V, B_{U}\right) \rightarrow \operatorname{Hom}(V, U) \rightarrow C_{V, U} \rightarrow 0
$$

has dimension $\delta_{[U],[V]}$. The module $\tau U$ is again indecomposable, and is called the Auslander-Reiten translate of $U$.

The Auslander-Reiten quiver $\Gamma_{Q}$ of $\bmod (\mathbb{K} Q)$ is defined as the quiver with vertices the isomorphism classes $[U]$ of indecomposables, and with an arrow $[U] \rightarrow[V]$ if there exists an irreducible map $f: U \rightarrow V$, that is, a nonzero non-isomorphism such that, in every factorization $f=h g$ for

$$
U \stackrel{g}{\rightarrow} X \stackrel{h}{\rightarrow} V
$$

either $g$ is a split monomorphism, or $h$ is a split epimorphism. Moreover, $\Gamma_{Q}$ has an additional "translation" structure with translation arrows $[U] \rightarrow[\tau U]$.

In fact, the vertices $[V]$ in $\Gamma_{Q}$ admitting an arrow to a non-projective $[U]$ (and, equivalently, those admitting an arrow from $[\tau U]$ ) can be characterized as the indecomposable direct summands of $B_{U}$.

The Auslander-Reiten quiver of $Q$ is known to be directed, which translates into the fact that the category $\bmod (\mathbb{K} Q)$ is representation-directed, which means that there 
exists an enumeration $\beta_{1}, \cdots, \beta_{N}$ of the positive roots such that

$$
\operatorname{Hom}\left(U_{\beta_{k}}, U_{\beta_{l}}\right)=0 \text { for } k>l \text { and } \operatorname{Ext}^{1}\left(U_{\beta_{k}}, U_{\beta_{l}}\right)=0 \text { for } k \leq l \text {. }
$$

We will write $U_{k}:=U_{\beta_{k}}$ for short. It is known [12] that there exists a sequence $i_{1}, \cdots, i_{N}$ in $I$ such that $\underline{w}_{0}:=s_{i_{1}} \cdots s_{i_{N}}$ is a reduced decomposition in $R\left(w_{0}\right)$, and such that $\beta_{k}=s_{i_{1}} \cdots s_{i_{k-1}}\left(\alpha_{i_{k}}\right)$ for all $k=1, \cdots, N$.

From this reduced decomposition, the Auslander-Reiten quiver can be recovered completely:

(1) $\Gamma_{Q}$ is isomorphic to the translation quiver defined in the following way:

- the vertices are $1,2, \cdots, N$;

- there are arrows $k \rightarrow \ell$ and $\ell \rightarrow k$ [1] (when $k$ [1] exists) if there exists an arrow $i_{\ell} \rightarrow i_{k}$ in $Q$, and $\ell$ is minimal among those indices larger than $k$ with this property;

- there is a translation $\ell \rightarrow k$ if $\ell=k[1]$.

(2) for $k \leq \ell$, the dimension $\left[U_{k}, U_{\ell}\right]$ equals $\left\langle\operatorname{dim} U_{k}, \operatorname{dim} U_{\ell}\right\rangle$;

(3) for $k \leq \ell$, the dimension $\left[U_{\ell}, U_{k}\right]^{1}$ equals $-\left\langle\operatorname{dim} U_{\ell}, \operatorname{dim} U_{k}\right\rangle$.

We define a partial order $U \preceq V$ on the indecomposable modules if there exists a path from $U$ to $V$ in the Auslander-Reiten quiver. By the above argument, this partial order is compatible with the directed enumeration, that is to say, if $U_{k} \preceq U_{\ell}$ then $k \leq \ell$.

\subsection{Hall algebras}

We recall the definition of the generic Hall algebra $\mathcal{H}(Q)$ of $Q$.

For representations $V, W$ and $X$ of $Q$ over a finite base field $\mathbb{F}_{q}$, we denote by $H_{V, W}^{X}$ the number of submodules $U$ of $X$ which are isomorphic to $W$, with quotient $X / U$ isomorphic to $V$. Now recall the field-independent parametrization of representations above; given functions $\varphi, \chi, \psi: \Delta_{+} \rightarrow \mathbb{N}$, we can then consider

$$
H_{\varphi, \chi}^{\psi}(q)=H_{M_{\varphi}\left(\mathbb{F}_{q}\right), M_{\chi}\left(\mathbb{F}_{q}\right)}^{M_{\psi}\left(\mathbb{F}_{q}\right)}
$$

as a function of the cardinalities $q$ of finite fields. In fact, it behaves polynomially in $q$ ([15]); thus, we will view $H_{\varphi, \chi}^{\psi}(q) \in \mathbb{Z}[q]$ as a polynomial in a variable $q$, called a Hall polynomial. By slight abuse of notation, we can then, for any field $\mathbb{K}$, define the Hall polynomial $H_{V, W}^{X}(q)$ by

$$
H_{V, W}^{X}(q)=H_{\varphi, \chi}^{\psi}(q) \text { if } V=M_{\varphi}(\mathbb{K}), W=M_{\chi}(\mathbb{K}), X=M_{\psi}(\mathbb{K}) .
$$

The generic Hall algebra $\mathcal{H}(Q)$ of $Q$ is then defined as the $\mathbb{Q}(q)$-algebra with linear $\mathbb{Q}(q)$-basis $F_{[V]}$ for $V$ ranging over all isomorphism classes in $\bmod (\mathbb{K} Q)$, with 
multiplication defined by:

$$
F_{[V]} F_{[W]}=\sum_{[X]} q^{[V, V]+[W, W]+\langle\operatorname{dim} V, \operatorname{dim} W\rangle-[X, X]} H_{V, W}^{X}\left(q^{2}\right) F_{[X]} .
$$

The most important fact about Hall algebras for our present purposes is that $\mathcal{H}(Q)$ is isomorphic to $U_{q}\left(\mathfrak{n}^{-}\right)$[15], the isomorphism

$$
\eta_{Q}: \mathcal{H}(Q) \rightarrow U_{q}\left(\mathfrak{n}^{-}\right)
$$

being given by $F_{\left[S_{i}\right]} \mapsto F_{i}$. This will now allow us to understand the LS formula in terms of quiver representations.

We first summarize some basic facts about Hall polynomials. For this, we assume the base field $\mathbb{K}$ to be algebraically closed from now on (to avoid some anomalies over small finite fields):

(1) $H_{V, W}^{X}(q) \neq 0$ if and only if there exists a short exact sequence $0 \rightarrow W \rightarrow X \rightarrow$ $V \rightarrow 0$.

(2) If $[W, V]=0$ and $X$ is an extension of $V$ by $W$, then $H_{V, W}^{X}(q)=1$.

(3) If $[V, W]=0$, then $H_{V, W}^{V \oplus W}(q)=q^{[W, V]}$.

For an indecomposable module $U$, we denote the image of $F_{[U]}$ under $\eta_{Q}$ by $F_{\operatorname{dim} U}$. Given a finite dimensional $\mathbb{K} Q$-module $M$, we can decompose it as

$$
M=\bigoplus_{k=1}^{N} U_{k}^{m_{k}}
$$

Then in the Hall algebra, we have

$$
F_{[M]}=F_{\left[U_{1}\right]}^{\left(m_{1}\right)} F_{\left[U_{2}\right]}^{\left(m_{2}\right)} \cdots F_{\left[U_{N}\right]}^{\left(m_{N}\right)}
$$

For two indecomposable modules $U \preceq V$ (and thus $[V, U]=0=[U, V]^{1}$ ), we define the $q$-commutator by:

$$
\left[F_{[V]}, F_{[U]}\right]_{q}:=F_{[V]} F_{[U]}-q^{[U, V]-[V, U]^{1}} F_{[U]} F_{[V]} .
$$

By the definition of the multiplication in the Hall algebra and the properties of the Hall polynomials mentioned above, we have

$$
F_{[U]} F_{[V]}=F_{[U \oplus V]}
$$

and

$$
F_{[V]} F_{[U]}=q^{[U, V]-[V, U]^{1}} F_{[U \oplus V]}+\sum_{[X]} c_{[X]} F_{[X]},
$$


the sum ranging over all isomorphism classes of non-split extensions $X$ of $V$ by $U$. We thus derive

$$
\left[F_{[V]}, F_{[U]}\right]_{q}=\sum_{[X]} c_{[X]} F_{[X]},
$$

the sum ranging again over all isomorphism classes of non-split extensions $X$ of $V$ by $U$. According to the first property of Hall polynomials above, all non-split extensions $X$ will appear on the right-hand side with nonzero coefficient $c_{[X]}$.

We note two special cases of such $q$-commutators:

(1) One special case is when $[V, U]^{1}=0$; then, $\left[F_{[V]}, F_{[U]}\right]_{q}=0$.

(2) The other special case is when $U=\tau V$ is the Auslander-Reiten translation of $V$. In this case, there exists exactly one non-split extension $X$ of $V$ by $U$ given by the middle term $B_{V}$ of the Auslander-Reiten sequence, and the $q$-commutator can be computed as

$$
\left[F_{[V]}, F_{[U]}\right]_{q}=\left(q-q^{-1}\right)^{[U, V]} F_{\left[B_{V}\right]}
$$

We translate these computations in $\mathcal{H}(Q)$ to $U_{q}\left(\mathfrak{n}^{-}\right)$using the algebra isomorphism $\eta_{Q}$ described above. Under this isomorphism, $F_{\left[U_{k}\right]}$ is sent to the quantum PBW root vector $F_{\beta_{k}}$ corresponding to the reduced decomposition arising from the enumeration of positive roots. Then the above formulae can be rewritten as

(1) If $\left(\beta_{\ell}, \beta_{k}\right)=0$, then $F_{\beta_{\ell}} F_{\beta_{k}}=F_{\beta_{k}} F_{\beta_{\ell}}$.

(2) If $1 \leq k<\ell \leq N$ and $\ell=k[1]$, then

$$
\left[F_{\beta_{\ell}}, F_{\beta_{k}}\right]_{q}=\left(q-q^{-1}\right)^{\left\langle\beta_{k}, \beta_{\ell}\right\rangle} \prod_{k<p<\ell,\left\langle\alpha_{i_{p}}, \alpha_{i_{k}}\right\rangle \neq 0} F_{\beta_{p}} .
$$

\subsection{Comparison of cones: quiver-compatible case}

We now introduce a $K$-theoretic cone associated with the quiver $Q$ and use the previous results to relate it to the quantum degree cone.

Recall the map $\operatorname{dim}: K_{0}^{\oplus} \rightarrow K_{0}$, and denote by $\Lambda$ its kernel. Then we have the short exact sequence of free abelian groups $0 \rightarrow \Lambda \rightarrow K_{0}^{\oplus} \rightarrow K_{0} \rightarrow 0$. Dualizing by taking $(-)^{*}:=\operatorname{Hom}(-, \mathbb{Z})$, we obtain a short exact sequence

$$
0 \rightarrow K_{0}^{*} \rightarrow\left(K_{0}^{\oplus}\right)^{*} \rightarrow \Lambda^{*} \rightarrow 0 .
$$

Note that, by definition, $\Lambda^{*}$ consists of additive functions on $K_{0}^{\oplus}$ modulo those functions depending only on the dimension vectors of modules.

Let $\Lambda_{\mathbb{R}}:=\Lambda \otimes_{\mathbb{Z}} \mathbb{R}$. We consider the cone $\mathcal{E} \subset \Lambda_{\mathbb{R}}$ generated by expressions $[V]+[W]-[X]$ whenever there exists a short exact sequence $0 \rightarrow V \rightarrow X \rightarrow W \rightarrow$ 0 . Inside $\mathcal{E}$, we consider the subcones:

(1) $\mathcal{E}^{\prime}$ generated by all $[V]+[W]-[X]$ for $V$ and $W$ indecomposable and $X$ an extension of $V$ by $W$, 
(2) $\mathcal{E}^{\prime \prime}$ generated by all $[U]+[\tau U]-\left[B_{U}\right]$ for $U$ a non-projective indecomposable.

Let $\mathcal{D}:=\mathcal{E}^{\vee} \subset \Lambda_{\mathbb{R}}^{*}$ be the dual cone of $\mathcal{E}$, that is,

$$
\mathcal{D}:=\left\{\varphi \in \Lambda_{\mathbb{R}}^{*} \mid \varphi(x) \geq 0 \text { for any } x \in \mathcal{E}\right\}
$$

The cone $\mathcal{D}$ thus consists of those functions $\varphi$ on $\Lambda_{\mathbb{R}}$ which are additive, that is, $\varphi([V \oplus W])=\varphi([V])+\varphi([W])$, and such that for any short exact sequence $0 \rightarrow$ $V \rightarrow X \rightarrow W \rightarrow 0$, we have $\varphi([X]) \leq \varphi([V])+\varphi([W])$.

Using the terminology of [9, Definition 2], such a function $\varphi$ is an admissible function on isomorphism classes of $\mathbb{K} Q$-modules. By [9, Theorem 4(1)], the cone $\mathcal{D}$ is freely generated by the functions $[U,-]$ for $U$ a non-projective indecomposable $\mathbb{K} Q$-module (since if $U$ is projective, then $[U,-]$ only depends on the dimension vector).

Lemma 3 We have $\mathcal{E}=\mathcal{E}^{\prime}=\mathcal{E}^{\prime \prime}$.

Proof By definition, we have inclusions $\mathcal{E}^{\prime \prime} \subset \mathcal{E}^{\prime} \subset \mathcal{E}$; thus, it suffices to prove that $\mathcal{E} \subset \mathcal{E}^{\prime \prime}$ or, in other words, that any expression $[V]+[W]-[X]$ can be written as a nonnegative linear combination of expressions $[U]+[\tau U]-\left[B_{U}\right]$. By [3], a linear combination

$$
[V]+[W]-[X]=\sum_{[U]} a_{[U]}\left([U]+[\tau U]-\left[B_{U}\right]\right)
$$

(the sum ranging over all non-projective indecomposables) with integral coefficients $a_{[U]}$ exists. For any non-projective indecomposable $Y$, applying $\operatorname{Hom}(Y,-)$ to all modules and using the defining property of almost split sequences, we find

$$
[Y, V]+[Y, W]-[Y, X]=a_{[Y]},
$$

which is thus nonnegative since $V, X$ and $W$ form a short exact sequence.

We can now prove the main result of this section:

Theorem 4 Let $Q$ be a quiver of type $\mathrm{A}, \mathrm{D}$ or $\mathrm{E}$ and $\underline{w}_{0} \in R\left(w_{0}\right)$ be compatible with Q. Then

$$
\mathcal{L}_{\underline{w}_{0}}^{-}\left(\mathfrak{g}^{L}\right)=\mathcal{D}_{\underline{w}_{0}}^{q}(\mathfrak{g})
$$

Proof Using $\mathcal{E}=\mathcal{E}^{\prime}$, we can characterize $\mathcal{D}$ as the cone of additive functions $\varphi$ on representations such that $\varphi([X]) \leq \varphi([V])+\varphi([W])$ whenever there exists a non-split short exact sequence $0 \rightarrow V \rightarrow X \rightarrow W \rightarrow 0$ with $V$ and $W$ indecomposable. Such modules can be written as $V=U_{k}, W=U_{\ell}$, for $k<\ell$, and $X=\bigoplus_{p} U_{p}^{n_{p}}$, where $n_{p} \neq 0$ only if $k<p<\ell$ (since otherwise, by directedness, there is no map from $U$ to $U_{p}$ or from $U_{p}$ to $V$ ). Using the quiver-theoretic interpretation of the LS formula 
developed above, $\mathcal{D}$ can thus be characterized as the cone of functions $\varphi$ on positive roots such that

$$
\varphi\left(\beta_{k}\right)+\varphi\left(\beta_{\ell}\right) \geq \sum_{k<p<\ell} n_{p} \varphi\left(\beta_{p}\right)
$$

whenever $F_{\beta_{k+1}}^{\left(n_{k+1}\right)} \cdot \ldots \cdot F_{\beta_{\ell-1}}^{\left(n_{\ell-1}\right)}$ appears with nonzero coefficient in the $q$-commutator $\left[F_{\beta_{\ell}}, F_{\beta_{k}}\right]_{q}$. But this is precisely the definition of the quantum degree cone $\mathcal{D}_{\underline{w}_{0}}^{q}(\mathfrak{g})$. On the other hand, using $\mathcal{E}=\mathcal{E}^{\prime \prime}$, we can characterize $\mathcal{D}$ as the cone of additive functions $\varphi$ on representations such that $\varphi\left(\left[B_{U}\right]\right) \leq \varphi([U])+\varphi([\tau U])$ for all non-projective indecomposables $U$. In this situation, we can write $\tau U=U_{k}, U=U_{k[1]}$, and we have

$$
B_{U}=\bigoplus_{\substack{k<p<k[1] \\ i_{p} \rightarrow i_{k}}} U_{p}
$$

by the combinatorial description of $\Gamma_{Q}$ stated above. Thus, $\mathcal{D}$ can be characterized as the cone of functions $\varphi$ on positive roots such that

$$
\varphi\left(\beta_{k}\right)+\varphi\left(\beta_{k[1]}\right) \geq-\sum_{k<p<k[1]} a_{i_{k}, i_{p}} \varphi\left(\beta_{p}\right),
$$

which is precisely the definition of the negative tight monomial cone $\mathcal{L}_{\underline{w}_{0}}^{-}(\mathfrak{g})$.

\subsection{Example}

Let $\mathfrak{g}=\mathfrak{s l}_{n+1}$ be of type $A_{n}$ with positive roots

$$
\Delta_{+}=\left\{\alpha_{i, j}=\alpha_{i}+\cdots+\alpha_{j} \mid 1 \leq i \leq j \leq n\right\}
$$

We compute the quantum degree cone $\mathcal{D}_{\underline{w}_{0}^{\max }}^{q}(\mathfrak{g})$ for

$$
\underline{w}_{0}^{\max }:=(n, n-1, n, n-2, n-1, n, \cdots, 1, \cdots, n) .
$$

This reduced decomposition is compatible with the following quiver of type $A_{n}$ :

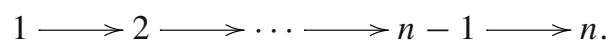

For simplicity, we denote $d_{i, j}:=d_{\alpha_{i, j}}$ for $1 \leq i \leq j \leq n$. By Theorem 4, it suffices to compute $\mathcal{L}_{\underline{w}_{0}^{\max }}^{-}\left(\mathfrak{g}^{L}\right)$, which is given by:

$$
\begin{aligned}
& \text { for } 1 \leq i \leq n-1, \quad d_{i, i+1} \leq d_{i, i}+d_{i+1, i+1} \\
& \quad \text { for } 1 \leq i<j-1 \leq n-1, \quad d_{i, j}+d_{i+1, j-1} \leq d_{i, j-1}+d_{i+1, j} .
\end{aligned}
$$




\section{Tropical flag varieties}

The quantum degree cone can be looked as a cone arising from non-commutative Gröbner theory. It is natural to ask for a connection to the (commutative) Gröbner theory of flag varieties. We describe such a connection in this section.

\subsection{Tropical varieties}

For an embedded projective variety $X \hookrightarrow \mathbb{P}^{N}$, the associated tropical variety with respect to its intersection with the standard torus in $\mathbb{P}^{N}$, roughly speaking, is a subfan in the Gröbner fan of the homogeneous prime ideal defining $X$ in $\mathbb{P}^{N}$. This polyhedral object is found to be very useful to derive and prove effective results in enumerative geometry and intersection theory (such as Gromov-Witten invariants). Maximal dimension cones in the tropical variety may give toric degenerations of $X$ (here a toric variety is not necessarily irreducible). We briefly recall the definition of a tropical variety in this setting, details see [4].

For a polynomial

$$
f:=\sum_{\mathbf{a} \in \mathbb{N}^{n+1}} \lambda_{\mathbf{a}} x^{\mathbf{a}} \in \mathbb{C}\left[x_{0}, x_{1}, \cdots, x_{n}\right],
$$

we define its support $\operatorname{supp}(f)$ as those tuples $\mathbf{a} \in \mathbb{N}^{n+1}$ such that $\lambda_{\mathbf{a}} \neq 0$. The tropicalization of $f$, denoted by $\operatorname{trop}(f)$, is a map from $\mathbb{R}^{n+1}$ to $\mathbb{R}$ defined by:

$$
\operatorname{trop}(f)(\mathbf{w}):=\min \{\mathbf{w} \cdot \mathbf{a} \mid \mathbf{a} \in \operatorname{supp}(f)\}
$$

Let $\mathbf{1}:=(1,1, \cdots, 1) \in \mathbb{R}^{n+1}$ and $H:=\mathbb{R}^{n+1} / \mathbb{R} \mathbf{1}$.

Let $I \subset \mathbb{C}\left[x_{0}, x_{1}, \cdots, x_{n}\right]$ be a homogeneous prime ideal and $V(I)$ be the (irreducible) projective variety associated with $I$. The tropical variety associated with $V(I)$ is defined by:

$\operatorname{trop}(V(I))=\bigcap_{f \in I}\{\mathbf{w} \in H \mid$ the minimum in $\operatorname{trop}(f)(\mathbf{w})$ is attained at least twice $\}$.

The tropical variety $\operatorname{trop}(V(I))$ is a polyhedral fan of $\operatorname{dimension} \operatorname{dim}(V(I))$; all vectors $\mathbf{v}$ in the relative interior of a cone $C$ give the same initial ideal in $C(I):=\operatorname{in}_{\mathbf{v}}(I)$; vectors in different cones give different initial ideals.

A cone $C$ in trop $(V(I))$ is called a maximal prime cone if $C$ is a cone of maximal dimension, and $\operatorname{in}_{C}(I)$ is a prime ideal. When $\operatorname{in}_{C}(I)$ is furthermore a binomial ideal, we get a flat degeneration of the variety $V(I)$ to the toric variety $V\left(\operatorname{in}_{C}(I)\right)$.

It is an important but hard question to give the ray descriptions or the facet descriptions of the cones in such a tropical variety. By the duality construction in [10], knowing the ray description of a maximal prime cone $C$ with binomial initial ideal allows one to recover a higher rank valuation on the homogeneous coordinate ring of $X:=\operatorname{Proj}(V(I))$ so that the associated Newton-Okounkov body is the polytope 
defining the toric variety $\operatorname{in}_{C}(I)$. On the other hand, we hope that having an explicit description to the facets may shed light on executing the algorithms in Gröbner theory, such as the Gröbner walk, to cross a codimension one face in order to obtain new reduced Gröbner bases, as well as the descriptions of the other cones [16].

\subsection{Tropical complete flag varieties}

We fix an integer $n>1$. Let $\mathrm{SL}_{n}$ be the special linear group defined over $\mathbb{C}$ and $B \subset \mathrm{SL}_{n}$ be the Borel subgroup consisting of upper triangular matrices in $\mathrm{SL}_{n}$. Let $\mathcal{F} l_{n}:=\mathrm{SL}_{n} / \mathrm{B}$ be the complete flag variety and $\mathbb{C}^{n}$ be the natural representation of $\mathrm{SL}_{n}$. We consider the Plücker embedding of $\mathcal{F} l_{n}$ into a product of projective spaces

$$
\mathcal{F} l_{n} \hookrightarrow \prod_{k=1}^{n-1} \mathbb{P}\left(\Lambda^{k} \mathbb{C}^{n}\right) .
$$

We fix the standard basis $e_{1}, \cdots, e_{n}$ of $\mathbb{C}^{n}$ and $I=\left\{i_{1}, \cdots, i_{k}\right\}$ such that $1 \leq i_{1}<$ $\cdots<i_{k} \leq n$. The linear function

$$
P_{I}:=\left(e_{i_{1}} \wedge e_{i_{2}} \wedge \cdots \wedge e_{i_{k}}\right)^{*} \in\left(\Lambda^{k} \mathbb{C}^{n}\right)^{*}
$$

is called a Plücker coordinate on the product of projective spaces.

For $I=\left\{i_{1}, \cdots, i_{s}\right\}, J=\left\{j_{1}, \cdots, j_{t}\right\}$ with $1 \leq t \leq s \leq n-1,1 \leq i_{1}<\cdots<$ $i_{s} \leq n, 1 \leq j_{1}<\cdots<j_{t} \leq n$ and a number $1 \leq k \leq t$, we define a Plücker relation

$$
R_{I, J}^{k}:=P_{I} P_{J}-\sum_{1 \leq p_{1}<\cdots<p_{k} \leq s} P_{I^{\prime}} P_{J^{\prime}}
$$

where

$$
P_{I^{\prime}}=\left(e_{i_{1}} \wedge \cdots \wedge e_{i_{p_{1}-1}} \wedge e_{j_{1}} \wedge e_{i_{p_{1}+1}} \wedge \cdots \wedge e_{i_{p_{2}-1}} \wedge e_{j_{2}} \wedge \cdots \wedge e_{i_{s}}\right)^{*}
$$

and

$$
P_{J^{\prime}}=\left(e_{i_{p_{1}}} \wedge \cdots \wedge e_{i_{p_{k}}} \wedge e_{j_{k+1}} \wedge \cdots \wedge e_{j_{t}}\right)^{*}
$$

Let $S_{n}$ be the free polynomial algebra in the Plücker coordinates $P_{I}$ with $1 \leq \# I \leq$ $n-1$ and $R_{n} \subset S_{n}$ the ideal generated by all possible Plücker relations $R_{I, J}^{k}$. It is a prime homogeneous ideal, and the complete flag variety $\mathcal{F} l_{n}$ is defined by $R_{n}$ in this product of projective spaces.

Let $\operatorname{trop}\left(\mathcal{F} l_{n}\right):=\operatorname{trop}\left(V\left(R_{n}\right)\right)$ be the tropical variety associated with the complete flag variety with respect to intersection with the standard torus in the Plücker embedding. Precisely, a point in $\operatorname{trop}\left(\mathcal{F} l_{n}\right)$ gives each Plücker coordinate a degree, such that the corresponding initial ideal contains no monomials. Further details and small dimension examples of $\operatorname{trop}\left(\mathcal{F} l_{n}\right)$ can be found in [4]. 


\subsection{From quantum degree cone to tropical flag variety}

The goal of this paragraph is to give the facet description of a cone in each tropical complete flag variety of type A.

We denote $[n]:=\{1,2, \cdots, n\}, S:=2^{[n]} \backslash\{\emptyset,[n]\}$ and recall that

$$
\underline{w}_{0}^{\max }:=(n-1, n-2, n-1, n-3, n-2, n-1, \cdots, 1, \cdots, n-1) .
$$

For $I=\left\{i_{1}, \cdots, i_{k}\right\} \in S$ with $i_{1}<\cdots<i_{k}$, we define two tuples $p_{1}<\cdots<p_{\ell}$ and $q_{\ell}<\cdots<q_{1}$ by:

$$
\left\{p_{1}, \cdots, p_{\ell}\right\}=[k] \backslash I, \quad\left\{q_{1}, \cdots, q_{\ell}\right\}=I \backslash[k] .
$$

Let $\mathfrak{g}=\mathfrak{s l}_{n}(\mathbb{C})$. We define a map

$$
\varphi: \mathcal{D}_{\underline{w}_{0}^{\max }}^{q}(\mathfrak{g}) \rightarrow \mathbb{R}^{S}
$$

by sending $\mathbf{d} \in \mathcal{D}_{\underline{w}_{0}^{\max }}^{q}(\mathfrak{g})$ to a vector $\varphi(\mathbf{d})$ whose coordinates $\varphi(\mathbf{d})_{I}$ are given by:

(1) if $I=[k]$ for some $1 \leq k \leq n-1, \varphi(\mathbf{d})_{I}:=0$;

(2) otherwise $\varphi(\mathbf{d})_{I}:=-\left(d_{p_{1}, q_{1}-1}+\cdots+d_{p_{\ell}, q_{\ell}-1}\right)$, where $d_{i, j}:=d_{\alpha_{i, j}}$.

The negative sign in the definition matches the definition of the initial ideal in the Gröbner theory which takes the maximal degree part in a polynomial.

Then one of the main theorems in [7] can be rephrased as:

Theorem 5 ([7]). The map $\varphi$ maps $\mathcal{D}_{\underline{w}_{0}^{\max }}^{q}(\mathfrak{g})$ bijectively onto the closure of a maximal cone in $\operatorname{trop}\left(\mathcal{F} l_{n}\right)$.

Remark 3 (1) The maximal prime cone obtained as the image of $\varphi$ gives the flat toric degeneration of $G / B$ to the toric variety associated with the Feigin-FourierLittelmann-Vinberg polytopes [7].

(2) It is shown in [14] that changing the directions of the inequalities in the example in Sect. 4.5 identifies other maximal prime cones in tropical flag varieties.

Example 1 The complete flag variety $\mathcal{F}_{3}$ is defined by $P_{12,1}^{1}:=P_{1} P_{23}-P_{2} P_{13}+$ $P_{3} P_{12}$ in $\mathbb{C}\left[P_{1}, P_{2}, P_{3}, P_{12}, P_{13}, P_{23}\right]$. The point $\mathbf{d}:=\left(d_{\alpha_{2}}, d_{\alpha_{1,2}}, d_{\alpha_{1}}\right)=(1,1,1)$ is in the quantum degree cone. The vector $\varphi(\mathbf{d})$ gives $P_{1}, P_{12}$ degree 0 and all other Plücker coordinates degree -1 . The initial part $\operatorname{in}_{\varphi(\mathbf{d})}\left(P_{12,1}^{1}\right)=P_{1} P_{23}+P_{3} P_{12}$ is a binomial ideal, implying that $\varphi(\mathbf{d}) \in \operatorname{trop}\left(\mathcal{F l}_{3}\right)$.

The mysterious part in the above theorem is the definition of the map $\varphi$ which is motivated by the work in [9]. In a forthcoming preprint, using the notion of birational sequences introduced in [8], we will give a general approach to define the map $\varphi$.

Acknowledgements X.F. would like to thank Gleb Koshevoy on discussions related to the tight monomial cones.

Funding Open Access funding enabled and organized by Projekt DEAL. 
Open Access This article is licensed under a Creative Commons Attribution 4.0 International License, which permits use, sharing, adaptation, distribution and reproduction in any medium or format, as long as you give appropriate credit to the original author(s) and the source, provide a link to the Creative Commons licence, and indicate if changes were made. The images or other third party material in this article are included in the article's Creative Commons licence, unless indicated otherwise in a credit line to the material. If material is not included in the article's Creative Commons licence and your intended use is not permitted by statutory regulation or exceeds the permitted use, you will need to obtain permission directly from the copyright holder. To view a copy of this licence, visit http://creativecommons.org/licenses/by/4.0/.

\section{References}

1. Assem, I., Skowronski, A., Simson, D.: Elements of the representation theory of associative algebras Techniques of representation theory London Mathematical Society Student Texts Vol. 1, vol. 65. Cambridge University Press, Cambridge (2006)

2. Backhaus, T., Fang, X., Fourier, G.: Degree cones and monomial bases of Lie algebras and quantum groups. Glasgow Math. J. 59(3), 595-621 (2017)

3. Butler, M.C.R.: Grothendieck groups and almost split sequences. In: Roggenkamp, K.W. (ed.) Integral Representations and Applications. Lecture Notes in Mathematics, vol. 882. Springer, Berlin (1981)

4. Bossinger, L., Lamboglia, S., Mincheva, K., Mohammadi, F Computing toric degenerations of flag varieties. In: Smith G., Sturmfels B. (eds) Combinatorial Algebraic Geometry. Fields Institute Communications, vol 80. Springer, New York, pp.247-281 (2017)

5. Caldero, P., Marsh, R., Morier-Genoud, S.: Represent. Theory. Realisation of Lusztig cones 8, 458-478 (2004)

6. de Concini, C., Kac, V.G.: Representations of quantum groups at roots of 1. Operator Algebras Unitary Representations, Enveloping Algebras and Invariant Theory. Progress in Mathematics 92, pp. 471-506. Boston-Basel-Berlin, Birkhäuser (1990)

7. Fang, X., Feigin, E., Fourier, G., Makhlin, I.: Weighted PBW degenerations and tropical flag varieties. Commun. Contemp. Math. 21(1), 1850016 (2019)

8. Fang, X., Fourier, G., Littelmann, P.: Essential bases and toric degenerations arising from birational sequences. Adv. Math 312(25), 107-149 (2017)

9. Fang, X., Fourier, G., Reineke, M.: PBW-Filtration on quantum groups of type $A_{n}$. J. Algebra 449, 321-345 (2016)

10. Kaveh, K., Manon, C.: Khovanskii bases higher rank valuations and tropical geometry. SIAM Journal on Applied Algebra and Geometry 3 (2): 292-336 (2019)

11. Levendorskii, S., Soibelman, Y.: Some applications of quantum Weyl groups. J. Geom. Phys. 7, 241254 (1990)

12. Lusztig, G.: Introduction to quantum groups. Reprint of the edition, p. 2010. Modern Birkhäuser Classics, Birkhäuser/Springer, New York (1994)

13. Lusztig, G.: Tight monomials in quantized enveloping algebras, In:Quantum deformations of algebras and their representations" ed. A.Joseph et al., Isr. Math. Conf. Proc. 7 (1993), Amer. Math. Soc, pp. 117-132

14. Makhlin, I.: Gelfand-Tsetlin degenerations of representations and flag varieties. Trans. Groups (2020). https://doi.org/10.1007/S00031-020-09622-z

15. Ringel, C.M.: Hall algebras and quantum groups. Invent. Math. 101, 583-592 (1990)

16. Sturmfels, B.: Gröbner bases and convex polytopes. University Lecture Series, vol. 8, p. xii+162. American Mathematical Society, Providence, RI (1996)

17. Xi, N.: A commutation formula for root vectors in quantized enveloping algebras. Pacific J. Math. 189(1), 179-199 (1999)

Publisher's Note Springer Nature remains neutral with regard to jurisdictional claims in published maps and institutional affiliations. 